\title{
Urbano e rural: famílias multi-instaladas, mobilidade e manejo dos recursos de várzea na Amazônia
}

Miguel Pinedo-Vasquez - Center for Environmental Research and Conservation, Columbia University.

Christine Padoch - Institute of Economic Botany, New York Botanical Garden.

Robin R. Sears - The School for Field Studies.

Eduardo S. Brondizio - Department of Anthropology and Anthropological Center for Training and Research on Global Environmental Change (ACT), Indiana University.

Peter Deadman - Department of Geography, University of Waterloo, Canada.

\section{Resumo}

Populações rurais na Amazônia, especialmente os ribeirinhos ou caboclos que vivem na grande região de várzea, mudam-se frequentemente. Por causa da efemeridade das margens dos cursos de água, assim como das áreas agrícolas e agroflorestais, e em função de incertezas fundiárias e de oportunidades para trabalho e mercados, em algumas regiões, em particular na região de várzea da bacia amazônica, famílias estão sempre prontas para a possibilidade da mudança, desmontando casas, abandonando comunidades e recomeçando sua vida em novas localidades. Neste breve artigo, apresentamos uma série de complexidades na história recente dos fluxos demográficos e das relações econômicas entre as zonas rurais e urbanas que tem caracterizado diferentes partes da bacia onde temos trabalhado, em particular as áreas estuarinas de Macapá e a várzea da Amazônia peruana.

\section{Palavras-Chave}

Famílias multilocalizadas, mobilidade, manejo de recursos naturais, Amazônia.

\begin{abstract}
The Amazon rural populations, especially the river people or the caboclos who live in the large region of meadow, are often moving from one place to another. Due to the fragility of the river edges of the water streams, as well as the agricultural and agro forest areas, and because of uncertainty of lands and opportunities for work and market, in some regions, particularly in the region of meadow of the Amazon basin, families are always ready for the possibility of moving, taking houses apart, leaving their communities and restart their lives in new different places. In this brief article, we present a series of complexities in the recent history of the demographic flows and of the economical relations between the rural and urban areas which have characterized different parts of the basin where we have worked, mainly the estuarine areas of Macapá and the meadow of the Peruvian Amazon.
\end{abstract}

\section{Keywords}

Multi-located families, mobility, management of natural resources, the Amazon region. 


\section{INTRODUÇÃO}

Populações rurais na Amazônia, especialmente os ribeirinhos ou caboclos que vivem na grande região de várzea, mudam-se frequentemente. A mobilidade das pessoas, a transitoriedade das comunidades e a mudança nas atividades econômicas na Amazônia têm sido mencionadas por muitos pesquisadores e documentadas em muitas comunidades no Brasil e Peru (ADAMS et al., 2006; BRONDIZIO, 2008; CASTRO 2006; CHIBNIK, 1994; NUGENT, 1993; PADOCH; JONG, 1990). Por causa da efemeridade das margens dos cursos de água, assim como das áreas agrícolas e agroflorestais, e em função de incertezas fundiárias e de oportunidades para trabalho e mercados, em algumas regiões, em particular na região de várzea da bacia amazônica, famílias estão sempre prontas para a possibilidade da mudança, desmontando casas, abandonando comunidades e recomeçando sua vida em novas localidades. Por exemplo, a comunidade de Santa Rosa no baixo rio Ucayali, fundada como um empreendimento agroextrativista ou fundo $0^{1}$ chamado Monte Carmelo no final da década de 20, tem-se reunido e dispersado repetidamente ao longo de sua história; mais recentemente, os santa-rosinos mudaram-se de uma margem do rio para a outra aproximadamente cinco vezes em um período de trinta anos (PADOCH; JONG, 1990; SANTOS-GRANERO; BARCLAY, 2000).

Embora os movimentos no campo não tenham cessado ao longo das últimas décadas, os fluxos migratórios na Amazônia têm trazido cada vez mais pessoas para as cidades. Como grande parte do mundo tropical, as áreas urbanas da região tiveram uma surpreendente taxa de crescimento. Em 1980, a maioria da Amazônia já era classificada com urbana (BECKER, 1985; WINKLERPRINS, 2002), mas, em 2000, o censo brasileiro (INSTITUTO BRASILEIRO DE GEOGRAFIA E ESTATÍSTICA, 2002) estimou que 70\% dos residentes da "Amazônia Legal" viviam em cidades (INSTITUTO DE PESQUISA ECONÔMICA APLICADA, 2002). Browder (2002) tem caracterizado o ritmo da urbanização da Amazônia brasileira, desde 1980, como "surpreendente".

As maiores cidades da Amazônia peruana, Iquitos e Pucallpa, também cresceram drasticamente a partir dos anos 60. Em grande parte devido à imigração maciça das zonas rurais das várzeas amazônicas do departamento de Loreto, a população de Iquitos aumentou mais que o quádruplo entre 1961 e 1993; no mesmo período de tempo, a população da cidade de Pucallpa, localizada ao longo da parte superior do rio Ucayali, aumentou mais de seis vezes (SANTOSGRANERO; BARCLAY, 2000).

A Amazônia é obviamente uma região vasta e diversa, cuja variabilidade

$1 \quad$ Fundo pode ser sítio ou fazenda em português. 
ambiental, econômica, política e demográfica leva à formação de diversos sistemas sociais e econômicos em zonas rurais e urbanas. Embora a urbanização maciça tenha sido uma tendência geral na região, essas mudanças não são simples, unidirecionais ou lineares. Neste breve artigo, apresentamos uma série de complexidades na história recente dos fluxos demográficos e das relações econômicas entre as zonas rurais e urbanas que tem caracterizado diferentes partes da bacia onde temos trabalhado, em particular as áreas estuarinas de Macapá e a várzea da Amazônia peruana. Começamos com uma breve análise demográfica do intercâmbio entre áreas rurais e urbanas nas últimas décadas, incluindo períodos nos quais se observam processos opostos às tendências observadas atualmente. Abordamos a formação e a importância atual de agregados domiciliares que caracterizamos como unidades domésticas "multilocalizadas", ou seja, aquelas que funcionam como unidades econômicas, mas possuem membros em zonas rurais e urbanas (PADOCH et al., 2008). Discutimos as implicações desses processos na formação de periferias urbanas e finalmente na gestão dos recursos nas várzeas do estuário e em outros locais (Figura 1).

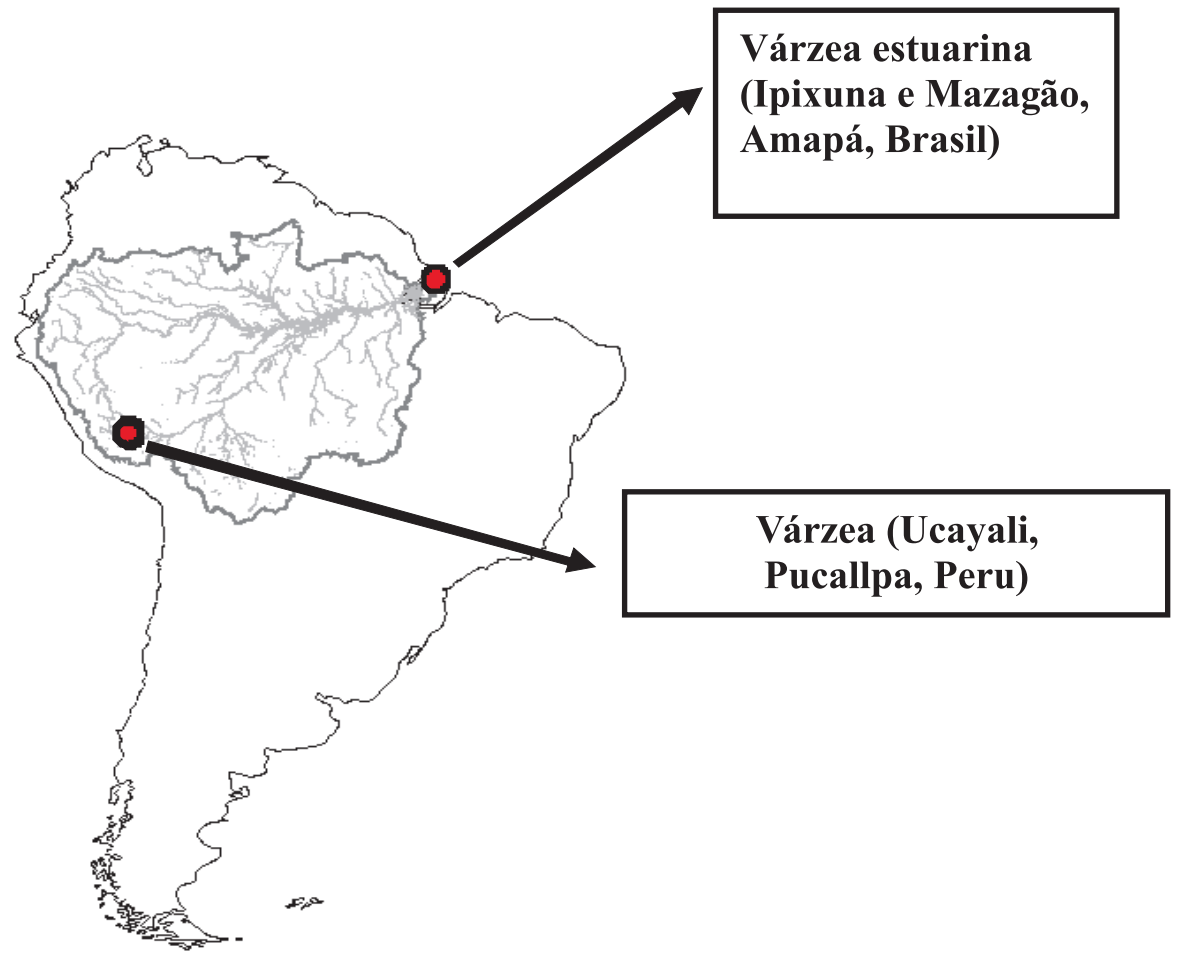

Figura 1: Regiões de estudo. 


\section{DO RURAL PARA O URBANO E VICE-VERSA NO ESTUÁRIO}

Embora as movimentações demográficas entre os setores rurais e urbanos da várzea do Amapá e de áreas vizinhas do Estado do Pará ainda não estejam bem documentadas (faltam números e datas exatos), nossa pesquisa preliminar revela que, nos anos 40 e no início dos anos 50, muitas vilas e cidades da região perderam até $50 \%$ de sua população devido à migração para zonas rurais. Naquele momento, particularmente durante a Segunda Guerra Mundial, florestas de estuário foram fontes de recursos para suprir a demanda e abastecer os mercados europeus com matérias-primas para sabão e outros produtos que dependiam dos recursos do Sudeste Asiático e de áreas do Pacífico ocupadas pelo exército japonês. Como em outras regiões de várzea amazônica, milhares de "soldados da borracha" penetraram a várzea para coletar borracha. Durante essas décadas, no Amapá e no Pará, os trabalhadores, incluindo muitos vindos de vilas e cidades, mudaram-se para as florestas de estuário para trabalhar na coleta e no processamento de óleo de palmeira, amêndoas e outros produtos florestais.

Esse movimento demográfico dramático de áreas urbanas para áreas rurais foi revertido duas décadas mais tarde, durante os anos 70. Naquela época, o incentivo à criação de búfalos nas ilhas do estuário causou um aumento na produção de carne e serviu para atender a demanda de países ricos em petróleo, como a Venezuela e a Arábia Saudita. Essa atividade aumentou o interesse pela compra de grandes extensões de terra na região. Isso resultou no deslocamento e na migração de habitantes rurais para as cidades. Áreas de várzea foram consideradas pelos especialistas como áreas altamente adequadas para a criação de búfalos, mas impróprias para a agricultura, embora muitas décadas e mesmo séculos de experiências agrícolas caboclas demonstrassem a produtividade potencial dessas áreas. Recursos do Estado e do governo federal foram usados para subsidiar a construção de casas na periferia das cidades, contribuindo para a recolocação de fazendeiros que perderam suas terras nas ilhas em outras regiões de várzea. Atualmente, em grande parte da região estuarina do Amapá e em certa parte vizinha do Estado do Pará, a população rural é 60\% menor do que em 1960, enquanto a população urbana aumentou mais de cinco vezes no mesmo período de tempo.

Esses números demonstram a variação no fluxo de pessoas que se movem entre as zonas urbanas e rurais. Podem, no entanto, dar uma impressão também enganosa das relações demográficas entre lugares rurais e urbanos. Embora, na Amazônia, as pessoas sejam classificadas simplesmente como residentes na zona urbana ou rural, pesquisadores afirmam que essas distinções não são simples e implicam relações de moradia e movimentos bem mais complicados. Aparentemente 
o habitante rural e o habitante urbano sempre foram, e continuam sendo atualmente, indistintos e indissoluvelmente ligados, em inúmeras formas (NUGENT, 1993; PADOCH et al., 2008; WAGLEY, 1953; WINKLERPRINS, 2002). Além do mais, as oscilações históricas nos padrões demográficos e econômicos, como mencionado acima, têm resultado em redes sociais e familiares complexas, que ainda são pouco compreendidas por políticos e cientistas sociais.

\section{URBANO E RURAL: FAMÍLIAS MULTILOCALIZADAS}

Nossa pesquisa sobre os migrantes de zonas rurais para zonas urbanas, nas cidades de Macapá e na vizinhança de Santana, revelou que muitas famílias pobres ou moradores de favela são realmente "multilocalizados", ou seja, mantêm uma casa e as atividades econômicas em uma área rural, mas também em uma das periferias ou favelas que rodeiam as cidades da região. Investigações preliminares sobre as fontes de renda mostram que uma percentagem considerável do rendimento familiar depende de recursos naturais, como o açaí (Euterpe oleracea Mart.), frutas, madeira, ou de outros produtos manejados ou extraídos em áreas rurais. Investigações em cinco comunidades rurais e perto das planícies aluviais do Amapá confirmam essas conclusões. A grande maioria das famílias de cinco comunidades tem uma casa em Macapá ou Santana (Tabela 1). Constatamos que todas as famílias que não possuem uma casa em uma área urbana eram compostas por casais muito jovens que tinham acabado de construir as suas casas na comunidade, com a ajuda de seus pais e de outros parentes. No entanto, todos esses jovens planejam construir uma residência na periferia de Macapá quando suas famílias crescerem.

Tabela 1: Famílias multilocalizadas em cinco aldeias no Amapá.

\begin{tabular}{|l|c|c|c|}
\hline \multicolumn{1}{|c|}{ Comunidade } & N. $^{\circ}$ Famílias & $\begin{array}{c}\text { N. }{ }^{\circ} \text { Famílias com } \\
\text { casa na cidade e na } \\
\text { comunidade }\end{array}$ & $\begin{array}{c}\text { N. }{ }^{\circ} \text { Famílias } \\
\text { com casa só na } \\
\text { comunidade }\end{array}$ \\
\hline Mazagão & 93 & 78 & 15 \\
Ipixuna & 81 & 63 & 18 \\
Bacabá & 78 & 62 & 16 \\
Santo Antônio & 101 & 93 & 8 \\
Lontra Pedreira & 126 & 106 & 20 \\
\hline
\end{tabular}

Famílias com residência na cidade e na comunidade ou multilocalizadas não são novidades para a Amazônia, mas esse processo tem-se intensificado nos 
últimos anos. Além das mudanças recentes nas comunicações e nos transportes, os mercados e as oportunidades de trabalho têm aumentado muito a incidência desse padrão residencial. Nugent (1993) descreveu várias dessas grandes famílias que viviam na região de Santarém e que se mantinham firmes em ambas as residências, na cidade e na comunidade, usando recursos de ambas. Mais recentemente, Winklerprins (2002) descreveu pequenos grupos ou famílias que mantinham duplos padrões de residência na região de Santarém. Esse padrão também caracteriza as comunidades rurais do Estado do Amapá. Residências múltiplas localizadas em toda a região estuarina são mais comumente compostas por grandes famílias, as quais mantêm uma casa na cidade e uma na comunidade ou no sítio na zona rural. A maioria dessas familias inclui um ou mais membros que tendem a permanecer na área urbana por mais tempo, enquanto outros membros circulam entre a comunidade e a cidade. Mudanças a curto e a longo prazos nas oportunidades de trabalho e na disposição dos recursos naturais e dos produtos agrícolas, bem como as alterações nas condições de mercado estimulam a contínua circulação de pessoas e de recursos entre países e cidades. Os sistemas familiares e as redes sociais em geral são fundamentais para a manutenção desse fluxo. O número exato dos residentes e a composição das famílias das zonas urbanas e rurais frequentemente variam com as safras agrícolas e com os horários escolares. Para as famílias da várzea, principalmente no Peru e em regiões como, por exemplo, o município de Tefé no Brasil, as variações na altura e na duração das enchentes anuais são outro determinante importante para residência.

\section{DO RURAL PARA O URBANO E PARA A PERIFERIA}

Transportes mais rápidos e fáceis e comunicações mais eficientes transformaram as múltiplas residências e as famílias multilocalizadas na Amazônia em um fenômeno comum em toda a região. Porém, entrevistando as famílias rurais que vêm das comunidades localizadas em tributários distantes da cidade, verificamos que é comum outro padrão mais complexo de moradia. Muitas famílias residentes nas periferias ou favelas que rodeiam Pucallpa, a segunda maior cidade da Amazônia peruana, mantêm não apenas dois locais residenciais, mas mesmo três. A terceira casa é geralmente construída em regiões suburbanas ou periurbanas onde estão localizados jardins, pomares e áreas de transformação de produtos agrícolas e florestais, destinados a atender as necessidades diárias dos membros das famílias urbanas; esses produtos incluem frutas e verduras, bem como alguns produtos comercializáveis. Outros produtos, como madeira, caça, peixe, muitas vezes provêm de locais distantes da base da família rural. Embora as razões econômicas 
sejam, sem dúvida, importantes para explicar tal dispersão, a preferência por morar na zona rural, mas com maior acesso a serviços urbanos, também é citada. Esse movimento tornou-se um incentivo para manter um fluxo das zonas rurais para as zonas urbanas e das zonas urbanas para as zonas rurais (Figura 2).

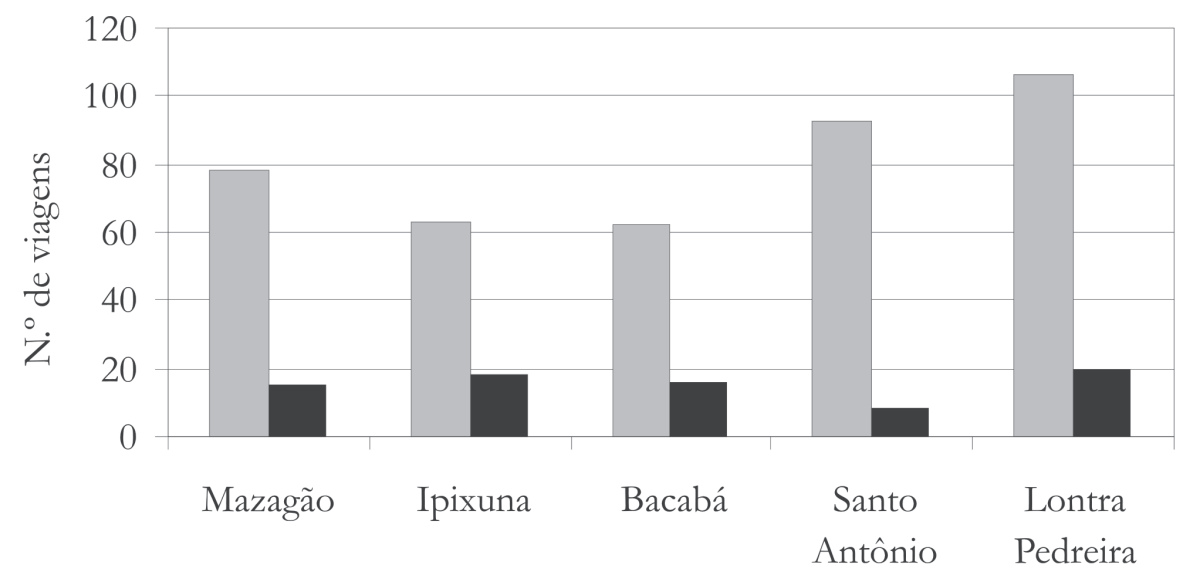

da zona rural para a zona urbana da zona urbana para a zona rural

Figura 2: Média anual do tempo de viagem entre as zonas rurais e urbanas de 50 famílias em 2006, 2007 e 2008

Esse estilo de vida suburbano é especialmente preferido pelos mais velhos, que gostam da vida rural e do trabalho agrícola e já dispõem de fácil acesso a serviços importantes, incluindo cuidados médicos, que são oferecidos, mesmo que precariamente, nas áreas periurbanas.

\section{DESAGRARIALIZAÇÃO DE ATIVIDADES E "RURALIZAÇÃO” DAS PREFERÊNCIAS}

Muitos dos padrões de residência que temos descrito na Amazônia coincidem com a tendência de desagrarialização das sociedades rurais que estão transformando a paisagem ao longo dos trópicos. A desagrarialização é geralmente entendida como um processo de diversificação nas atividades econômicas e nas fontes de rendimento, bem como uma mudança na identificação social dos produtores agrícolas. Segundo os pesquisadores, o agricultor familiar está cada vez mais praticando atividades não agrícolas (BRYCESON, 1996; ELLIS, 1998). Essa tendência pode ser verificada em escala mundial. Por exemplo, uma análise das mudanças ocorridas na África subsaariana concluiu que, no final dos anos 90, de $60 \%$ a $80 \%$ dos rendimentos das famílias rurais provinham de fontes não agrícolas, 
enquanto na década de 80 essas fontes eram responsáveis por aproximadamente $40 \%$ de seus rendimentos (BRYCESON, 1999). O deslocamento das áreas rurais para as áreas urbanas em geral implica mudanças no modo de vida (DE HAAN, 1999), assim como a redução e a recomposição das comunidades rurais.

Embora a maioria dos estudos sobre desagrarialização tenham sido feitos na África subsaariana e no Sudeste Asiático (BARRETT; REARDON; WEBB, 2001; BOOTH, 2002; BRYCESON, 1996, 1999; RIGG; NATTAPOOLWAT, 2001), processos similares podem ser observados ao longo das várzeas amazônicas, como a diversidade de ocupação que acompanha a mobilidade rural-urbano e a formação de unidades domésticas multilocalizadas (NUGENT, 1993; WINKLERPRINS, 2002). A investigação feita por Pinedo-Vasquez e outros (2001), em comunidades de várzea do Amapá, tem recentemente documentado uma forte tendência para a desagrarialização. A agricultura como fonte de renda tem diminuído, e a maior parte das famílias em assentamentos rurais depende da liquidez gerada pelos membros que não só vivem, mas também trabalham em zonas urbanas, como Macapá e Santana, além de contarem com auxílios governamentais (como o Programa Bolsa Família) e aposentadorias. As zonas rurais e urbanas representam a base da economia familiar, contribuindo assim com uma variedade de recursos e uma rede de suporte social. Esses tópicos ainda necessitam de uma investigação mais precisa para se compreender o conteúdo, o volume e a direção desses fluxos de recursos e suas respectivas redes sociais e econômicas. Entretanto, os recursos que circulam dentro das famílias com múltiplas instalações não estão confinados simplesmente aos rios, às florestas e à produção agrícola. Importantes recursos urbanos que são trocados incluem novas tecnologias de produção, trabalho, ferramentas, artigos específicos de consumo e capital. Além disso, esses membros ampliam a rede social familiar para diferentes cidades, para empresas onde podem estar empregados e, não menos, para agências governamentais e/ou organizações não governamentais $(\mathrm{ONG})$ que facilitam o acesso à representação política e a recursos econômicos.

A utilização dos solos, os padrões de produção e de consumo dessas famílias com instalações residenciais múltiplas refletem uma combinação de necessidades e oportunidades rurais e urbanas. Por exemplo, Brondizio e seus colegas descobriram que a atividade econômica mais importante da região estuarina hoje, a produção do açaí, foi desenvolvida como resposta a um aumento na demanda do alimento devido à migração, após 1970, de residentes rurais que trouxeram para a zona urbana o consumo de açaí como comida básica (BRONDIZIO, 2004, 2008). Nesse caso, o consumo de açaí nas grandes cidades representa o deslocamento de uma preferência alimentar - com pequenas alterações nos gostos e nas formas - das 
áreas rurais para áreas urbanas, aliado ao baixo custo e à disponibilidade do produto. As famílias residentes nas favelas ou periferias de Macapá, em Belém e em outros centros urbanos continuam a manter laços e a compartilhar gostos com o mundo circundante da zona rural, embora também adquiram novas preferências urbanas. A recente propagação do consumo de açaí nas populações urbanas sem experiência rural pode ser vista com uma forma de "ruralização" de gosto e padrão de consumo no rápido crescimento das cidades amazônicas e para além delas. Entretanto, esses processos têm mudado com a popularização nacional e internacional do açaí e o consequente aumento dos preços, principalmente nos períodos de entressafra.

Em nossa pesquisa anterior, observamos que as famílias do Amapá cujos membros trabalharam para serrarias de grande porte em áreas urbanas aplicam a experiência e o conhecimento adquiridos sobre o processamento de madeira à produção doméstica, além de incorporarem novas técnicas de manejo florestal a suas atividades rurais (PINEDO-VASQUEZ et al. 2001; SEARS; PADOCH; PINEDO-VASQUEZ, 2007). Essas famílias, que possuem residências múltiplas, têm montado serrarias familiares e atualmente fornecem uma grande variedade de produtos madeireiros baratos a um mercado urbano crescente. Essas famílias também podem ser favorecidas com o grande número de espécies que são localmente utilizadas para a construção. Enquanto as indústrias de madeira orientadas para a exportação e baseadas em madeira de lei do estuário amazônico tendem a processar e a comercializar somente seis espécies, a nova indústria madeireira rural/urbana usa mais de 45 espécies de madeiras pesadas e leves, muitas delas produzidas em sistemas agroflorestais. Com base na aquisição de experiências urbanas e rurais, as famílias multilocalizadas na várzea do Amapá têm desenvolvido novos padrões de produção de madeira, de transformação e de comercialização, atingindo, assim, o mercado de construção em Macapá, bem como em outras cidades amazônicas. Atualmente, uma parte das grandes indústrias madeireiras do estuário tem sido substituída por produção de pequena escala, de administração familiar, e por práticas florestais diversificadas e industriais. Esses fluxos não lineares e multidirecionais de pessoas e outros capitais em famílias rurais e urbanas, que variam em forma e conteúdo em diferentes partes da região, até hoje continuam a ser pouco estudados.

\section{ORGANIZAÇÕES E REDES URBANAS E RURAIS}

Nosso trabalho tem enfatizado a importância da análise de unidades familiares e suas relações de parentesco para a compreensão das ligações entre o urbano 
e o rural na Amazônia. Existem, no entanto, outros tipos de redes sociais e organizações governamentais e não governamentais que têm historicamente ajudado a mobilizar e a mover recursos, trabalho e capital, além de apoiarem famílias em zonas tanto rurais como urbanas. Embora essas instituições urbanas/rurais sejam muito variáveis em tamanho, composição e estrutura, elas tendem a ser flexíveis em suas funções e são amplamente conectadas. Entre essas organizações que operam em diversas partes da região, incluem-se vários tipos de associações, sindicatos de trabalhadores rurais e outras formas de união sindical (e.g., união dos trabalhadores do Amapá), que, por meio de ações e petições aos órgãos governamentais, têm sido de valor inestimável para ajudar os agricultores a ter acesso a capital e a recursos nas suas zonas rurais e também para ajudar famílias nas suas negociações relativas a questões urbanas. Um exemplo de instituição inovadora são as Escolas Família, que ensinam jovens da zona rural em diversas áreas do Brasil (incluindo as comunidades estuarinas do Amapá), oferecendo-lhes uma formação profissional relevante no contexto em que vivem. Embora algumas dessas escolas sejam acusadas de manter jovens membros de famílias agrícolas nas zonas rurais (negando-lhes oportunidades urbanas), o ensino que oferecem pode, de fato, ajudá-los a lidar mais eficazmente com as necessidades tanto rurais como urbanas, promovendo, assim, mesmo que de maneira indireta, a formação de famílias multilocalizadas, como temos observado. Em contrapartida, organizações e redes sociais na Amazônia peruana incluem "comitês municipais", grupos organizados no âmbito provincial e distrital que têm por objetivo ajudar a identificar famílias rurais que necessitam de apoio e de acesso a programas para enfrentar os desafios do ambiente periurbano e urbano, muitas vezes desconhecido. No Peru, grupos semelhantes, em âmbito ainda mais local, incluem organizaciones vecinales ou "organizações de bairro", que também ajudam habitantes rurais a desenvolver oportunidades, como a oferecida pelo programa WAWASI, que se concentra especificamente na ajuda às mulheres, incluindo as mães solteiras. Essas organizações auxiliam famílias a ter acesso a seguro saúde, assistência jurídica, crédito e serviços, bem como a obter empregos temporários nas cidades.

Cidades e vilas amazônicas também apresentam em maior ou menor grau uma variedade de associações comunitárias que promovem a ligação entre imigrantes de comunidades rurais específicas, ajudando-os a manter e a reforçar os laços entre o rural e o urbano. Por exemplo, a Asociación de Dos de Mainos (Associação da População de Dos de Mayo), que congrega imigrantes na cidade de Pucallpa do rio Ucayali, distante da aldeia Dos de Mayo, tem sido fundamental para ajudar os recém-chegados a lidar com habitação, emprego, governo e vários processos de licenciamento; é também fundamental para a manutenção 
e o desenvolvimento de laços econômicos e sociais entre o rural e o urbano. A associação trabalha fortemente ligando os novos imigrantes àqueles que chegaram primeiro e que são relativamente mais bem-sucedidos, bem relacionados e/ou trabalham em posições-chave e em instituições públicas e privadas na cidade. Por exemplo, uma enfermeira que é membro do grupo tem desempenhado um papel importante, ajudando as pessoas da associação a encontrar serviços de saúde adequados na zona urbana. Organizações sociais similares têm apoiado imigrantes em todo o mundo, auxiliando-os, em muitos períodos, a serem assimilados nas suas novas localidades, sem cortar as ligações com as suas áreas de origem.

Apresentamos neste artigo apenas algumas das muitas "complexidades" do movimento urbano-rural ao longo das últimas décadas na Amazônia. Essas relações tendem a crescer e a alcançar um escala cada vez mais importante na Amazônia. Essa pesquisa demorou a ser elaborada, mas novos estudos serão realizados à medida que se acelere a urbanização da bacia. O presente estudo oferece uma descrição geral dos processos que ajudarão a definir o futuro rural/ urbano ou urbano/rural da Amazônia. Deve-se lembrar, contudo, que, enquanto processos de urbanização, desagrarialização, multilocalização e mesmo periurbanização podem crescer drasticamente, essas tendências não são simples, unidirecionais, nem lineares, nem resultam das recentes formas de "globalização".

\section{REFERÊNCIAS}

ADAMS, C.; MURRIETA, R. S. S.; NEVES, W. A. (Ed.). Sociedades caboclas amazốnicas: modernidade e invisibilidade. São Paulo: FAPESP; Annablume, 2006.

BARRETT, C. B.; REARDON, T.; WEBB, P. (Ed.). Income Diversification and Livelihoods in Rural Africa: Cause and Consequence of Change. Food Policy, v. 26, n. 4, p. 315-453, ago. 2001. Número especial.

BECKER, B. K. Fronteira e urbanização repensadas. Revista Brasileira de Geografia, v. 47, n. 3/4, p. 357-371, 1985.

BOOTH, A. The changing role of non-farm activities in agricultural households in Indonesia: some insights from the agricultural censuses. Bulletin of Indonesian Economic Studies, v. 38, n. 2, p. 179-200, 2002.

BRONDIZIO, E. S. From staple to fashion food: shifting cycles, shifting opportunities and the case of Açaí fruit (Euterpe oleracea Mart.) of the Amazon estuary. In: ZARIN, D. (Ed.). Working forests of the American tropics: management for sustainable forest? New York: Columbia University Press, 2004. p. 123-137.

BRONDIZIO, E. S. The Amazonian Caboclo and the Açaipalm: forest farmers in the 
global market. New York: New York Botanical Garden Press, 2008.

BROWDER, J. The urban-rural interface: urbanization and tropical forest cover change. Urban Ecosystem, v. 6, n. 1/2, p. 21-41, 2002.

BRYCESON, D. F. Deagrarianization and rural employment in sub-Saharan Africa: A sectoral perspective. World Development, v. 24, n. 1, p. 97-111, jan. 1996.

BRYCESON, D. F. African rural labour, income diversification and livelihood approaches: a long-term development perspective. Review of African Political Economy, v. 26, n. 80, p. 171-189, 1999.

CASTRO, E. M. R. Belém de águas e ilhas. 1. ed. Belém: CEJUP, 2006.

CHIBNIK, M. Risky rivers: the economics and politics of floodplain farming in Amazonia. Tuczon: University of Arizona Press, 1994.

DE HAAN, A. Livelihoods and poverty: the role of migration - a critical review of the migration literature. Journal of Development Studies, v. 36, n. 2, p. 1-47, 1999.

ELLIS, F. Household strategies and rural livelihood diversification. Journal of Development Studies, v. 35, n. 1, p. 1-38, 1998.

INSTITUTO BRASILEIRO DE GEOGRAFIA E ESTATÍSTICA. Tabulação avançada do Censo Demográfico 2000: resultados preliminares da amostra. Rio de Janeiro, 2002.

INSTITUTO DE PESQUISA ECONÔMICA APLICADA. Caracterização e tendências da rede urbana do Brasil: redes urbanas regionais: Norte, Nordeste e CentroOeste. Brasília, DF, 2002.

NUGENT, S. Amazonian caboclo society: an essay on invisibility and peasant economy. Providence: Berg Publishers, 1993.

PADOCH, C.; JONG, W. de. Santa Rosa: the impact of the forest products trade on an Amazonian place and population. In: PRANCE, G. T.; BALICK, M. J. (Ed.). New directions in the study of plants and people: advances in Economic Botany. New York: The New York Botanical Garden, 1990, v. 8. p. 151-158.

PADOCH, C.; E. BRONDIZIO; COSTA, S.; PINEDO-VASQUEZ, M.; SEAR, R. R.; SIQUEIRA, A. Urban Forest and Rural Cities: Multi-sited Households, Consumption Patterns, and Forest Resources in Amazonia. Ecology and Society, v.13, n.2, p.2, 2008.

PINEDO-VASQUEZ, M.; ZARIN, D.; COFFEY, K.; PADOCH, C.; RABELO, F. Post-boom timber production in Amazonia. Human Ecology, v. 29, n. 2, p. 219- 
239, jun. 2001.

RIGG, J.; NATTAPOOLWAT, S. Embracing the global in Thailand: activism and pragmatism in an era of deagrarianization. World Development, v. 29, n. 6, p. 945-960, jun. 2001.

SANTOS-GRANERO, F; BARCLAY, F. Tamed frontiers: society, and civil rights in upper Amazonia. Washington, DC: Westview Press, 2000.

SEARS, R. R.; PADOCH, C.; PINEDO-VASQUEZ, M. Amazon forestry transformed: integrating knowledge for smallholder timber management in Eastern Brazil. Human Ecology, v. 35, n. 6, p. 697-707, 2007.

WAGLEY, C. Amazon town: a study of man in the Tropics. Oxford: Oxford University Press, 1953.

WINKLERPRINS, A. M. G. A. House-lot gardens in Santarém, Pará, Brazil: linking rural with urban. Urban Ecosystems, v. 6, n. 1/2, p. 43-65, 2002. 


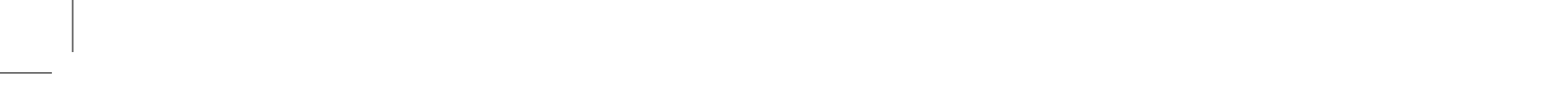

